\title{
Vibrational Power Flow Analysis of a Cylindrical Shell Using a Four-Point Technique
}

\author{
H. Salimi-Mofrad \\ Department of Mechanical Engineering, IOOC, Tehran, Iran.
}

\author{
S. Ziaei-Rad and M. Moradi \\ Department of Mechanical Engineering, Isfahan University of Technology, Isfahan, Iran.
}

\begin{abstract}
(Received 20 October 2012; provisionally accepted 31 March 2013; accepted 25 April 2013)
The aim of studying and analysing vibrational characteristics of structures using power flow is to control the noise and vibration within the structure and prevent it from being transmitted into the environment. The power flow within a cylindrical shell is investigated because of its importance in designing spacecraft and marine structures. In this paper, a four-point power flow technique was used to examine the effects of flexural and shear forces on the total power flow in a cylindrical shell in free-free conditions. To obtain better results, the exponential window was used considering the use of a hammer for the excitation of the structure. The examining of the results and obtained diagrams determined that the effect of shear forces on the total power flow was more than the effect of flexural forces. Moreover, the precision of two-point and four-point techniques was compared.
\end{abstract}

\section{INTRODUCTION}

Nowadays, the attention of many researchers and scholars in the field of vibration science is focused on energy reduction entered from a source, which generates noise to a structure and impedes its circulation into the environment. The Power Flow method is deemed proper for the measurement of vibrational energy in the structure. This method is applied as well in determining noise production source position within the structure. Vibration intensity is defined in rigid bodies and its measurement is performed at the level of structure. The reason for this logic comes from the fact that in thin structures, wave propagation within the structure is normally estimated in a definite way by its propagation in the structure. ${ }^{1}$

Several studies have been conducted on the cylindrical shell. One was made by Noisex. He discovered that the peak power flow of a structure happens in its vibrational mode. ${ }^{2}$ Fuller and Fahy inspected the free wave propagation in cylindrical shells. They studied the physical interpretation of wave propagation equations in a coupled system. ${ }^{3}$ Langley tested the frequency modification effect and changes of material specifications on the power flow. ${ }^{4}$ Zhang investigated the input power flow effect on the shells. He used different external forces and frequency during his study. ${ }^{5}$ In his investigation, a cylindrical shell filled infinitely with the fluid was considered. Fang proved that the effect of coupling of the fluid and shell on the response is important. ${ }^{6}$

Another problem to be solved was evaluating energy propagation in an arbitrary thin-walled structure yet unsolved. ${ }^{7}$ For the purpose of studying the vibrational behaviour of thin shells, many techniques were developed and applied. Arnold and Warburton applied an energy method and used Lagrange's equation as well as Love's first approximation theory. They studied the free vibration of a thin cylindrical shell with freely supported ends. ${ }^{8}$ Lam and Loy applied beam functions as the axial modal functions in the Ritz process for examining boundary condition effects on the free vibration characteristics for a cylindrical shell which was layered with nine different boundary conditions. ${ }^{9}$

Zhang and White inspected the power input of a shell due to point force excitation. A good degree of correlation was observed by comparing the driving and transfer points' test acceleration in the frequency range of study with the theoretical results. ${ }^{10}$ Merkulov et al. merely tested the point force excitation of an infinite thin-walled cylindrical shell filled with fluid. ${ }^{11}$ Energy flow through an arbitrary cross-section of an infinitely long shell was formulated by Sorokin at various circumferential mode numbers. During other studies performed, the inspected issue was "the energy redistribution among several transmission routes in a near-field and the influence of excitation conditions on steady fluctuations of the overall energy flow in a far field."12

In a study examining the dynamic characteristics of cylindrical shells, Heckl obtained the input impedance of a simplysupported cylindrical shell; however, the analysis was not covering the influence of the bending stiffness of the shell. His study had restrictions in estimating input power in practical applications. ${ }^{13}$ Harari offered a general formula, which assessed the loss of transmitted energy or power based on the structural impedance of finite and semi-infinite cylindrical shells.14 Missaoui et al. performed another study on the free and forced vibrations of a cylindrical shell with a floor partition, according to variational formulation. In this study, the structural coupling was simulated using artificial spring systems. ${ }^{15}$ As for describing structural coupling between the shell and plate, $\mathrm{Li}$ et al. applied artificial stiffness, presenting a methodology on the structural acoustic coupling characteristics of a cylindrical shell with an internal floor partition. ${ }^{16}$

Wang and Xing investigated the dynamic characteristics of power flow in a coupled plate-cylindrical shell system. ${ }^{17}$ Mandal and his colleagues applied the two-transducer method for naturally-orthotropic plates, in order to estimate vibration energy transfer in technically-orthotropic plates. They concluded that the higher the rigidity of the plate, the lower the vibration energy transmission. As the rigidity of the plates increases, the vibration amplitudes of it decrease, thereby reducing acoustic radiation. ${ }^{18}$ Zhu et al. have also studied the vibrational power flow of a thin cylindrical shell with a circumferential surface crack. They showed that changes obviously depend on 